\title{
Efeito de ondas curtas por método indutivo na lombalgia crônica inespecífica em indivíduos sedentários
}

\author{
Effect of inductive shortwave on chronic non-specific low back pain \\ in sedentary individuals
}

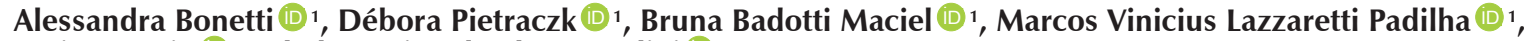 \\ Taciane Stein $\left(\mathbb{D}^{2}\right.$, Gladson Ricardo Flor Bertolini $\mathbb{D D}^{2} \bowtie$ \\ ' Universidade Estadual do Oeste do Paraná. Cascavel, PR, Brasil. \\ Universidade Estadual do Oeste do Paraná, Programa de Pós-Graduação em Biociências e Saúde. Cascavel, PR, Brasil.
}

Como citar este artigo (How to cite this article):

Bonetti A, Pietraczk D, Maciel BB, Padilha MVL, Stein T, Bertolini GRF. Efeito de ondas curtas por método indutivo na lombalgia crônica inespecífica em indivíduos sedentários (Effect of inductive shortwave on chronic non-specific low back pain in sedentary individuals). Sci Med. 2018;28(4):ID31670. DOI: 10.15448/1980-6108.2018.4.31670

\section{RESUMO}

OBJETIVOS: Avaliar a eficácia do tratamento com ondas curtas por método indutivo em indivíduos sedentários com lombalgia crônica inespecífica.

MÉTODOS: Um ensaio clínico quase-experimental e cruzado foi realizado com indivíduos lombálgicos e sedentários. A amostra foi recrutada entre acadêmicos da Universidade Estadual do Oeste do Paraná (Unioeste) na faixa etária entre 18 e 25 anos, que apresentavam dor lombar de origem postural não traumática há mais de três meses, eram sedentários, e aceitaram participar da pesquisa. O protocolo de intervenção consistiu na aplicação de ondas curtas por método indutivo por 15 minutos, uma vez ao dia, três vezes por semana, com intervalo de um dia entre cada aplicação. Na semana seguinte os voluntários recebiam tratamento placebo também por três vezes na semana, com tempo semelhante àquele realizado na semana tratamento, porém o equipamento era apenas ligado, sem emissão do campo eletromagnético. O protocolo foi aplicado por duas semanas. As variáveis analisadas foram o grau de incapacidade funcional, avaliado pelo Índice de Incapacidade de Oswestry (ODI) e pelo Questionário de Incapacidade de Roland-Morris (QIRM), no início do experimento e ao final de cada semana; e a dor, avaliada pela Escala Visual Analógica (EVA), antes e após cada sessão. Os dados foram apresentados em média e desvio-padrão ou mediana e quartis e o nível de significância aceito foi de $5 \%$.

RESULTADOS: Vinte voluntários participaram do estudo. Para a ODI houve redução dos valores de incapacidade ao longo da semana de tratamento, com retorno aos valores iniciais na semana placebo; já para o QIRM não houve diferenças entre o tratamento e o placebo. Pela EVA, houve redução na intensidade da dor ao longo dos três dias de terapia, fato que ocorreu apenas no segundo dia do placebo.

CONCLUSÕES: O tratamento com ondas curtas na modalidade indutiva foi eficaz na diminuição da dor em pacientes sedentários com lombalgia crônica, contribuindo para melhora da capacidade funcional.

DESCRITORES: ondas curtas; ondas de rádio; medição da dor; dor lombar; diatermia.

\section{ABSTRACT}

AIMS: To evaluate the efficacy of inductive shortwave treatment in sedentary individuals with non-specific chronic low back pain.

METHODS: A quasi-experimental and cross-over trial was performed with sedentary individuals with chronic back pain. The sample was recruited among students from the State University of Western Paraná (Unioeste), aged between 18 and 25 years old, who had low back pain of non-traumatic postural origin for more than three months, were sedentary, and accepted to participate in the study. The intervention protocol consisted in the application of short waves by inductive method for 15 minutes, once a day, three times a week, with interval of one day between each application. In the following week, volunteers also received placebo treatment three times a week, with time similar to that performed in the treatment week, but the equipment was only switched on, without emission of the electromagnetic field. The protocol was applied for two weeks. The variables analyzed were the degree of functional disability, assessed by the Oswestry Disability Index (ODI) and the Roland-Morris Disability Questionnaire (QIRM), at the beginning of the experiment and at the end of each week; and pain, assessed by the Visual Analogue Scale (VAS), before and after each session. Data were presented as mean and standard deviation or median and quartiles, and the accepted level of significance was $5 \%$.

RESULTS: Twenty volunteers participated in the study. For ODI, there was a reduction in the disability values throughout the treatment week, with a return to previous values in the placebo week; there were no differences between treatment and placebo for the MIRR. By VAS, there was a reduction in pain intensity over the three days of therapy, which occurred only on the second day of the placebo.

CONCLUSIONS: Treatment with short waves in the inductive modality was effective in reducing pain in sedentary patients with chronic low back pain, contributing to improve functional capacity.

KEYWORDS: short waves; radio waves; pain measurement; low back pain; diathermy. 
Abreviaturas: EVA, Escala Visual Analógica de Dor; ODI, Índice de Incapacidade de Oswestry (Oswestry Disability Index); QIRM, Questionário de Incapacidade de Roland-Morris.

\section{INTRODUÇÃO}

A lombalgia é um quadro de alta incidência e prevalência. Estima-se que aproximadamente $80 \%$ da população apresentem ao menos um episódio de lombalgia em algum momento de suas vidas. É considerada crônica aquela com duração entre sete e 12 semanas, sendo os sintomas mais relevantes a dor e a incapacidade funcional [1-6]. O tratamento para condições agudas e subagudas consiste em repouso relativo, adaptações nas atividades de vida diária, anti-inflamatórios não hormonais, manipulação e tratamento fisioterapêutico. Em casos crônicos, são utilizados também relaxantes musculares, antidepressivos e opioides, contudo, tais formas de tratamento costumam apresentar efeitos colaterais $[7,8]$; por isso, modalidades fisioterapêuticas como terapia manual [9], eletroestimulação [10], frio, calor, exercício, ultrassom, terapia manual, são opções utilizadas, apesar da falta de evidências científicas [7].

Campos eletromagnéticos produzem calor em tecidos biológicos por induzir um movimento alternante em íons, rotação de moléculas dipolares e distorção de moléculas não polares. A movimentação iônica é devida a um fluxo de corrente e ocorre em tecidos ricos em eletrólitos, tais como vasos sanguíneos e músculos, sendo que a resistência a este fluxo gera calor. Equipamentos comerciais com frequência de $27,12 \mathrm{MHz}$ e comprimentos de onda de $11,06 \mathrm{~m}$, seguindo padrões internacionais, são denominados de ondas curtas, pertencendo a bandas de rádio. São equipamentos projetados para emissão de ondas de forma contínua ou pulsada, visando aquecer tecidos profundos [11]. Segundo a lei de Van't Hoff, ocorre aumento na taxa de reações químicas com o aumento na temperatura, acelerando o metabolismo celular, o consumo de oxigênio e o gasto energético. Aumentos na taxa metabólica causados por diatermia irão acelerar o processo de reparo e inflamação, e acrescidos da vasodilatação local, ocasionam melhora na drenagem e retirada de catabólitos, podendo auxiliar no reparo tecidual e na redução da dor $[8,11]$.

A diatermia com ondas curtas tem sido utilizada há diversas décadas, com finalidades analgésicas e como facilitadora do reparo tecidual [8]. Contudo, existem controvérsias com relação aos efeitos da mesma, e pobreza na literatura com relação ao seu uso em pacientes com dor lombar crônica [12-17]. Assim, o objetivo do presente estudo foi avaliar a eficácia de ondas curtas por método indutivo na lombalgia crônica inespecífica em indivíduos sedentários.

\section{MÉTODOS}

A presente pesquisa caracteriza-se como um ensaio clínico quase-experimental e cruzado. A amostra foi constituída de indivíduos lombálgicos e sedentários, e recrutada entre acadêmicos da Universidade Estadual do Oeste do Paraná (Unioeste). O projeto de pesquisa foi aprovado pelo Comitê de Ética em Pesquisa da Unioeste, sob parecer 2.252.233. Todos os voluntários assinaram o termo de consentimento livre e esclarecido prévio ao início das avaliações.

Para participar do estudo, os indivíduos deveriam atender aos seguintes critérios de inclusão: ausência de alterações cognitivas, quadro de dor lombar de origem postural não traumática há mais de três meses, sedentarismo, faixa etária entre 18 e 25 anos e concordância em participar da pesquisa. Foram considerados sedentários indivíduos que não realizavam periodicamente exercícios físicos. Os critérios de exclusão foram: marca-passo cardíaco; um diagnóstico específico, como hérnia, espondilolistese, etc., ou radiculopatias associadas; sínteses metálicas; alteração da sensibilidade térmica; gravidez ou período menstrual; tecidos isquêmicos; doença maligna; trombose venosa profunda; ferida cutânea aberta; epilepsia; e faltar em alguma das sessões.

Para o cálculo de tamanho de amostra foram utilizados diferença mínima entre as médias de 2,0, desvio padrão de 1,7 , poder de $80 \%$ e significância de $5 \%$, resultando em 20 indivíduos. Os convites para os participantes foram feitos por meio de mídias sociais e contato direto, até obter 20 voluntários ( 3 homens e 17 mulheres).

O protocolo de intervenção consistiu na aplicação de ondas curtas por método indutivo por 15 minutos, uma vez ao dia, três vezes por semana, com intervalo de um dia entre cada aplicação. Na semana seguinte os voluntários recebiam tratamento placebo também por três vezes na semana, com tempo semelhante àquele realizado na semana tratamento, porém o equipamento era apenas ligado, sem emissão do campo eletromagnético. Dessa forma o experimento durou duas semanas, sendo considerados dois grupos (tratamento e placebo), porém ambos compostos pelos mesmos indivíduos, em momentos e com procedimentos diferentes. 
O aparelho de ondas curtas utilizado foi o Thermopulse $\left(\right.$ Ibramed $^{\mathbb{\circledR}}$ ), com frequência de $27,12 \mathrm{MHz} \pm 0,6 \%$. Foi utilizada a forma indutiva, com área de placa de $85 \mathrm{~cm}^{2}$. O aplicador indutivo foi conectado em contato direto com o paciente sobre a região lombar e fixado com faixa elástica. Para isso o paciente permanecia durante a terapia em decúbito ventral, com apoio na região abdominal, visando reduzir a lordose fisiológica. A dose utilizada foi baseada na classificada como grau 3 na escala de Schilephake, ou seja, um calor agradável. O mesmo terapeuta foi responsável durante todo o experimento, não sendo cegado com relação ao uso efetivo ou não do equipamento.

As variáveis analisadas foram o grau de incapacidade funcional, avaliado pelo Índice de Incapacidade de Oswestry (ODI - Oswestry Disability Index) e pelo Questionário de Incapacidade de Roland-Morris (QIRM), no início e final de cada semana; e a dor, avaliada pela Escala Visual Analógica de Dor (EVA), antes e após cada sessão.

O ODI é usado para avaliação funcional da coluna lombar, incorporando questões sobre a dor e atividade física [18]. Consiste em 10 questões com seis alternativas, variando de 0 a 5 . A primeira pergunta avalia a intensidade da dor, e as outras nove perguntas avaliam o efeito da dor sobre as atividades diárias: cuidados pessoais, elevar pesos, caminhar, sentar, dormir, posição ortostática, vida sexual e social. O escore total é dividido pelo número 50 (máximo escore possível) e multiplicado pelo número 100 , obtendo assim a porcentagem que define o nível de incapacidade. O ODI é classificado em incapacidade mínima (0 a 20\%), incapacidade moderada (21 a 40\%), incapacidade severa (41 a 60\%), invalidez (61 a 80\%), e indivíduo restrito ao leito ( 81 a $100 \%)$. O tempo necessário para sua aplicação é em geral de 10 minutos [18].

Outro instrumento de avaliação utilizado, o QIRM, possui 24 itens com pontuações de 0 ou 1 (sim ou não) e o total varia de 0 (sugerindo nenhuma incapacidade) a 24 (incapacidade grave). O QIRM possui boa validade e confiabilidade para medida de incapacidade física em pacientes com dor crônica. Seu tempo de aplicação é de aproximadamente 5 minutos [19]. Ambas as formas de avaliação funcional foram realizadas previamente ao primeiro dia de tratamento, ao final da primeira semana (tratamento) e ao final da segunda semana (placebo), sendo que um único avaliador foi responsável pelo uso desses instrumentos.

A intensidade da dor foi quantificada, antes e depois de cada sessão, por meio da Escala Visual Analógica (EVA), usando um dispositivo de madeira com um cursor metálico, numerado de 0 (ausência de dor) a
10 (intensidade máxima de dor), sendo solicitado ao voluntário indicar com o cursor o nível de intensidade de sua dor. $\mathrm{Na}$ face oposta à apresentada ao paciente há uma escala de $10 \mathrm{~cm}$, apresentada apenas ao avaliador, que dessa forma conseguia aferir a resposta do paciente. Essa forma de avaliação foi realizada em todos os momentos pré e pós-terapia, por um avaliador exclusivo para esta variável.

Para a análise estatística foi utilizado o programa Bioestat 5.0. Os dados foram apresentados em média e desvio-padrão para a EVA, com uso de ANOVA unidirecional e pós-teste de Bonferroni. Para o ODI e o QIRM os dados foram apresentados em mediana e quartis e comparados pelo teste de Friedman. Em todos os casos o nível de significância aceito foi de 5\%.

\section{RESULTADOS}

Os resultados obtidos pelo ODI apontaram que durante a semana de tratamento com ondas curtas houve redução nos escores de incapacidade, e na semana placebo a incapacidade voltou a subir de forma significativa, indicando efeitos da terapêutica adotada. Já para o QIRM os valores apontaram que houve uma redução na incapacidade funcional para as duas semanas seguintes ao início do tratamento e do placebo, sem diferença entre essas avaliações, apontando que o QIRM não detectou efeito importante do uso de ondas curtas (Tabela 1).

Tabela 1. Resultados do Índice de Incapacidade de Oswestry e do Questionário de Incapacidade de Roland-Morris para os voluntários em seu momento basal (antes do início da terapia), tratados com ondas curtas (final da $1 \underline{a}$ semana) e submetidos ao procedimento placebo (final da $2 \underline{\text { a }}$ semana).

\begin{tabular}{lccc}
\hline & Pré-tratamento & Ondas Curtas & Placebo \\
\hline ODI & & & \\
Mediana & 16 & $10^{*}$ & $15^{\#}$ \\
10 e 3o quartis & $12-18$ & $7,5-12$ & $10-16,5$ \\
QIRM & & & \\
Mediana & 5 & $3^{*}$ & $4^{*}$ \\
1ํ e 3o quartis & $2,7-4.85$ & $1,37-3,45$ & $1,92-4,1$ \\
\hline
\end{tabular}

ODI, Índice de Incapacidade de Oswestry; QIRM, Questionário de Incapacidade de Roland-Morris

* Diferença significativa ao comparar com o momento pré-tratamento $(p<0,05)$.

\# Diferença significativa ao comparar com o momento pós-tratamento com ondas curtas $(\mathrm{p}<0,05)$.

Os resultados obtidos pela EVA apontaram para redução da intensidade da dor nos três dias de terapia com ondas curtas, fato que ocorreu com o grupo placebo apenas no segundo dia. Quando comparados os dois grupos (tratamento e placebo), no momento prévio ao procedimento não houve diferenças entre eles, mas 
Tabela 2. Dados da Escala Visual Analógica (EVA) para os momentos pré e pós-procedimento, na primeira semana (tratamento) e na segunda semana (placebo).

\begin{tabular}{|c|c|c|c|c|c|c|}
\hline & \multicolumn{2}{|c|}{$10 \mathrm{dia}$} & \multicolumn{2}{|c|}{$2^{o}$ dia } & \multicolumn{2}{|c|}{$3^{\circ}$ dia } \\
\hline & Pré & Pós & Pré & Pós & Pré & Pós \\
\hline \multicolumn{7}{|l|}{ Ondas curtas } \\
\hline Média \pm DP & $5,9 \pm 1,9$ & $1,0 \pm 1,3^{*}$ & $4,0 \pm 2,1$ & $0,8 \pm 1,2^{*}$ & $5,6 \pm 1,8$ & $0,95 \pm 1,4^{*}$ \\
\hline \multicolumn{7}{|l|}{ Placebo } \\
\hline Média $\pm \mathrm{DP}$ & $5,5 \pm 2,2$ & $3,8 \pm 1,8^{\#}$ & $5,8 \pm 2,0$ & $0,9 \pm 1,7^{*}$ & $5,7 \pm 2,1$ & $4,6 \pm 2,0^{\#}$ \\
\hline
\end{tabular}

Pré, antes do procedimento; Pós, depois do procedimento; DP, desvio padrão.

* Diferença significativa ao comparar com o momento prévio à terapia $(p<0,05)$.

\#Diferença significativa ao avaliar o mesmo momento de terapia, nos diferentes grupos $(p<0,05)$.

para o momento após o procedimento, no primeiro e terceiro dia, houve diferenças, sendo que os valores da intensidade da dor (em centímetros) observados para o grupo tratamento foram menores do que os encontrados para o grupo placebo, apontando efeitos na redução do quadro doloroso com o tratamento de ondas curtas (Tabela 2).

\section{DISCUSSÃO}

A dor crônica é um importante sinal para os cuidados à saúde, visto o impacto negativo que causa sobre a qualidade de vida, e também os custos financeiros envolvidos [8]. A dor lombar crônica produz incapacidade funcional, impactando atividades laborais, da vida diária e outras [19].

Com respeito ao equipamento de ondas curtas há resultados controversos sobre seus efeitos terapêuticos em lesões musculoesqueléticas, visando tanto efeitos analgésicos como pró-reparo. Estudos experimentais apontaram resultados positivos na condroproteção [20], no reparo muscular [21], assim como melhora clínica em pacientes com osteoartrite de joelho [14]. Outros estudos não apontaram vantagens na osteoartrite [13], bem como na lesão muscular induzida por contração excêntrica [22]. Contudo, há consenso de que a indução de calor profundo pode interferir com os tecidos corporais [23].

Segundo Coelho et al. [18] o ODI apresenta uma boa correlação e consistência interna com o QIRM. A escala de Oswestry permite uma melhor avaliação nos pacientes com sérios problemas, enquanto que a de Roland-Morris quantifica melhor pacientes menos incapacitados. No presente estudo, pode-se aferir que os indivíduos possuíam incapacidade mínima, porém, na comparação entre os grupos, de forma contrária ao supracitado, apenas o ODI mostrou diferenças entre os dois grupos, sendo que o QIRM apontou melhora funcional tanto para os voluntários atendidos com ondas curtas quanto placebo. Uma possível explicação para essa diferença pode ter sido o pequeno prazo de tratamento e também da reavaliação, o que dificultaria a percepção das alterações funcionais.

A EVA, apesar de avaliar a dor em apenas uma dimensão, é um instrumento muito utilizado em pesquisa sobre dor. Neste estudo, apontou diferenças significativas entre os momentos pré e pós-terapia nos três dias em que foi realizado o tratamento com ondas curtas. O calor agradável, que foi a sensação referida pelos voluntários, produziu redução do quadro álgico, corroborando com os achados de Ahmed et al. [12] que, em estudo com delineamento semelhante ao presente, trataram pacientes com lombalgia crônica com antiinflamatórios não hormonais, exercícios e orientações, associados ou não a ondas curtas (utilizando grupo placebo), ao longo de seis semanas. Os autores observaram melhoras tanto para o grupo que recebeu quanto para o que não fez uso do calor, mas para o grupo que recebeu as ondas curtas os resultados foram significativamente melhores [12].

A presente amostra foi composta por estudantes de graduação, que em geral passam mais de quatro horas diárias sentados, muitas vezes de forma inadequada, o que colabora para distúrbios osteomusculares que podem levar à lombalgia. Para essa população, o calor pode auxiliar na redução da dor por ação direta sobre a musculatura esquelética, bem como por aumento de fluxo sanguíneo local, auxiliando na remoção de catabólitos acumulados [8].

Uma limitação do presente estudo foi o tipo de amostra estudada, que apresentava mínima limitação funcional. Entretanto, ressalta-se que há pobreza na literatura sobre os efeitos da diatermia de ondas curtas com método indutivo em lombalgia crônica não específica, de forma que os achados do presente estudo trazem contribuições, que devem ser aprofundadas 
com novas pesquisas envolvendo indivíduos com maior disfunção, assim como com outras doses da terapia utilizada. Podemos concluir que os resultados encontrados sugerem que a intervenção com ondas curtas indutivas foi eficaz na diminuição da dor em pacientes sedentários com lombalgia crônica, contribuindo para melhora na capacidade funcional.

\section{NOTAS}

Apoio financeiro

Este estudo não recebeu apoio financeiro de fontes externas.
Declaração de conflito de interesses

Os autores declaram não haver conflitos de interesses relevantes ao conteúdo deste estudo.

Contribuições dos autores

Todos os autores fizeram contribuições substanciais para concepção, ou delineamento, ou aquisição, ou análise ou interpretação de dados; e redação do trabalho ou revisão crítica; e aprovação final da versão para publicação.

Disponibilidade dos dados e responsabilidade pelos resultados

Todos os autores declaram ter tido total acesso aos dados obtidos e assumem completa responsabilidade pela integridade destes resultados.

\section{REFERÊNCIAS}

1. de Figueiredo VF, Pereira LSM, Ferreira PH, Pereira AM, de Amorim JSC. Incapacidade funcional, sintomas depressivos e dor lombar em idosos. Fisioter em Mov. 2013;26(3):549-57. https://doi.org/10.1590/S0103-51502013000300008

2. Gotfryd AO, Valesin Filho ES, Viola DCM, Lenza M, da Silva JA, Emi AS, Tomiosso R, Piccinato CA, Antonioli E, Ferretti M. Analysis of epidemiology, lifestyle, and psychosocial factors in patients with back pain admitted to an orthopedic emergency unit. Einstein (São Paulo). 2015;13(2):243-8. https://doi.org/10.1590/S1679-45082015 AO3320

3. da Silva MC, Fassa AG, Valle NCJ. Dor lombar crônica em uma população adulta do Sul do Brasil: prevalência e fatores associados. Chronic low back pain in a Southern Brazilian adult population: prevalence and associated factors. Cad Saúde Pública. 2004;20(2):377-85. https://doi.org/10.1590/S0102-311X2004000200005

4. Furtado RNV, Ribeiro LH, de Arruda AB, Descio FJ, Martucci Junior CE, Serruya DC. Dor lombar inespecífica em adultos jovens: fatores de risco associados. Rev Bras Reumatol. 2014;54(5):371-7. https://doi.org/10.1016/j.rbr.2014. 03.018

5. Garcia JBS, Hernandez-Castro JJ, Nunez RG, Pazos MAR, Aguirre JO, Jreige A, Delgado W, Serpentegui M, Berenguel M, Cantisani AF. Prevalence of low back pain in Latin America: a systematic literature review. Pain Physician. 2014;17(5):379-91.

6. Hoy D, Bain C, Williams G, March L, Brooks P, Blyth F, Woolf A, Vos T, Buchbinder R. A systematic review of the global prevalence of low back pain. Arthritis Rheum. 012;64(6):2028-37. https://doi.org/10.1002/art.34347

7. Patrick N, Emanski E, Knaub MA. Acute and chronic low back pain. Med Clin North Am. 2016;100(1):169-81. https:// doi.org/10.1016/j.mcna.2015.08.015

8. Rawe IM. The case for over-the-counter shortwave therapy: safe and effective devices for pain management. Pain Manag. 2014;4(1):37-43. https://doi.org/10.2217/pmt.13.60

9. Hughes CM, Quinn F, Baxter GD. Complementary and alternative medicine: Perception and use by physiotherapists in the management of low back pain. Complement Ther Med. 2011;19(3):149-54. https://doi.org/10.1016/j.ctim.2011. 03.003

10. Johnston C, Paglioli EB, Paglioli EB. Escore funcional e de dor após cirurgia de hérnia de disco lombar e fisioterapia precoce. Sci Med. 2006;16(4):151-6.

11. Goats GC. Continuous short-wave (radio-frequency) diathermy. Br J Sports Med. 1989;23(2):123-7. https://doi. org/10.1136/bjsm.23.2.123

12. Ahmed S, Shakoor A, Khan AA. Evaluation of the effects of shortwave diathermy in patients with chronic low back pain. Bangladesh Med Res Counc Bull. 2009;35(1):18-20. https://doi.org/10.3329/bmrcb.v35i1.2320

13. Akyol Y, Durmus D, Alayli G, Tander B, Bek Y, Canturk F, Sakarya T. Does short-wave diathermy increase the effectiveness of isokinetic exercise on pain, function, knee muscle strength, quality of life, and depression in the patients with knee osteoarthritis? A randomized controlled clinical study. Eur J Phys Rehabil Med. 2010;46(3):325-36.

14. Atamaz FC, Durmaz B, Baydar M, Demircioglu OY, Iyiyapici A, Kuran B, Oncel S, Sendur OF. Comparison of the efficacy of transcutaneous electrical nerve stimulation, interferential currents, and shortwave diathermy in knee osteoarthritis: A double-blind, randomized, controlled, multicenter study. Arch Phys Med Rehabil. 2012;93(5):748-56. https://doi. org/10.1016/j.apmr.2011.11.037

15. Dziedzic K, Hill J, Lewis M, Sim J, Daniels J, Hay EM. Effectiveness of manual therapy or pulsed shortwave diathermy in addition to advice and exercise for neck disorders: A pragmatic randomized controlled trial in physical therapy clinics. Arthritis Care Res. 2005;53(2):214-22. https://doi.org/10.1002/art.21087 
16. Kaysin MY, Akpinar P, Aktas I, Ozkan FU, Karamanlioglu DS, Hartevioglu HC, Vural N. Effectiveness of shortwave diathermy for subacromial impingement syndrome and value of night pain for patient selection: a double-blinded, randomized, placebo-controlled trial. Am J Phys Med Rehabil. 2018;97(3):178-86. https://doi.org/10.1097/PHM. 0000000000000819

17. Rattanachaiyanont MMD, Kuptniratsaikul VMD. No additional benefit of shortwave diathermy over exercise program for knee osteoarthritis in peri-/post-menopausal women: an equivalence trial. Osteoarthr Cartil. 2008;16(7):823-8. https:// doi.org/10.1016/j.joca.2007.10.013

18. Falavigna A, Teles AR, De Braga GL, Barazzetti DO, Lazzaretti L, Tregnago AC. Instrumentos de avaliação clínica e funcional em cirurgia da coluna vertebral. Coluna/Columna. 2011;10(1):62-7. https://doi.org/10.1590/S180818512011000100012

19. Coelho RA, Siqueira FB, Ferreira PH, Ferreira ML. Responsiveness of the Brazilian-Portuguese version of the Oswestry Disability Index in subjects with low back pain. Eur Spine J. 2008;17(8):1101-6. https://doi.org/10.1007/s00586-0080690-1

20. Ungur RA, Florea A, Tăbăran AF, Scurtu IC, Onac I, Borda IM, Irsay L, Ciortea VM, Dronca E, Zdrenghea MT, Suciu SM. Chondroprotective effects of pulsed shortwave therapy in rabbits with experimental osteoarthritis. Rom J Morphol Embryol. 2017;58(2):465-72.

21. Lideo L, Milan R. Ultrasound monitoring of shortwave diathermic treatment of gastrocnemius strain in a dog. J Ultrasound. 2013;16(4):231-4. https://doi.org/10.1007/s40477-013-0044-7

22. Castellani JW, Zambraski EJ, Sawka MN, Urso ML. Does high muscle temperature accentuate skeletal muscle injury from eccentric exercise? Physiol Rep. 2016;4(9):1-11. https://doi.org/10.14814/phy2.12777

23. Draper DO, Hawkes AR, Johnson AW, Diede MT, Rigby JH. Muscle heating with megapulse II shortwave diathermy and rebound diathermy. J Athl Train. 2013;48(4):477-82. https://doi.org/10.4085/1062-6050-48.3.01 